\title{
ON NILPOTENT AND SOLVABLE MALCEV ALGEBRAS
}

\author{
ERNEST L. STITZINGER
}

\begin{abstract}
Solvable and nilpotent Malcev algebras are investigated in the spirit of extending results from Lie algebras. The first section presents a proof of Engel's theorem with the Jacobson refinement and some consequences. The second part deals with conjugacy of Cartan subalgebras and consequences.
\end{abstract}

In his recent paper on Frattini subalgebras, A. A. el Malek observes that Cartan subalgebras exist in solvable Malcev algebras. He mentions that a technique used in the Lie algebra case by D. W. Barnes carries over to the present setting. A purpose of this note is to investigate the behavior of Cartan subalgebras in solvable Malcev algebras. A main tool is Engel's theorem for Malcev algebras. The existence of this important result is known, but a complete proof is not easily found in the literature. Moreover, it is not stated under the conditions used by Jacobson in his refinement of Engel's theorem in the Lie algebra case (see [8, p. 33]). Hence, a second objective of this paper is to prove Engel's theorem with the Jacobson refinement and to investiage some consequences which are extensions of results due to Jacobson and to Barnes in the Lie case.

Background material on the theory of Malcev algebras is found in the fundamental papers of Sagle [10] and Kuzmin [9].

1. Engel's theorem. Recall that a Malcev algebra $A$ is a nonassociative algebra which satisfies $x^{2}=0$ and $(x y)(t z)=((t y) z) x+((y z) x) t+((z x) t) y+((x t) y) z$. The algebras in this paper will be finite dimensional over a field of characteristic not 2. For $x \in A, R_{x}$ will denote the right multiplication. If $M$ is a vector space, a representation of $A$ on $M$ is a linear mapping $S: A \rightarrow \mathcal{L}(M)$ such that $S_{(x y) z}=S_{x} S_{y} S_{z}-S_{z} S_{x} S_{y}-S_{y z} S_{x}+S_{y} S_{z x}$ and $M$ is called an $A$-module. We consider these modules to be two sided under the rule $m a=-a m$. Let $X$ be a subset of $A . M X^{[n]}$ will denote the set of all $\left(\ldots\left(m x_{1}\right) \ldots\right) x_{n}$ where $m \in M$ and $x_{i} \in X$. Also let $M X^{[0]}=M . X$ is said to act nilpotently on $M$ if there exists an integer $n$ such that $M X^{[n]}=0$. For the smallest such $n$, there exists $z \in M X^{[n-1]}$, $z \neq 0$ but $z X=0$. Note that $X$ acts nilpotently on $M$ if and only if its linear span also acts nilpotently on $M$. A multiplicative set in $A$ is a subset of $A$ which is closed under multiplication. We now turn to a Malcev algebra version of Engel's theorem with the Jacobson refinement $[8$, p. 33]. It should be noted that in the Lie algebra case it is not assumed that the module is faithful. An interesting formula due to A. A. el Malek [4, Lemma 2.1] will be used in the proof.

THEOREM 1 (ENGEL'S THEOREM). Let $A$ be a Malcev algebra, $M$ a faithful $A$-module and $U$ a multiplicative set in $A$ which spans $A$. Suppose that $S_{x}$ is

Received by the editors August 16, 1983 and, in revised form, October 25, 1983.

1980 Mathematics Subject Classification. Primary 17A30. 
nilpotent for each $x \in U$ where $S$ is the representation of $A$ on $M$. Then $A$ acts nilpotently on $M$ and $A$ is nilpotent.

ProOF. We begin by showing that the assumptions translate to the $A$-module $B=M+A$. Let $m>4 \operatorname{dim} M$. Let $z \in M, x \in A$ and $y \in U$. By Lemma 2.1 of $[\mathbf{4}], z S_{\left(x y^{m+1}\right)}=h(m) z\left[S_{\left(x y^{m}\right)}, S_{y}\right]$ where $h(m)$ is a scalar and $x y^{m}$ stands for $x\left(R_{y}\right)^{m}$. By repeated applications of this result

$$
z S_{\left(x y^{m+1}\right)}=\sum_{i+k=m+1-j}^{k=m+1-j} C_{k, j, m} z\left(S_{y}\right)^{i} S_{\left(x y^{j}\right)}\left(S_{y}\right)^{k}
$$

for fixed $j \geq 2 \operatorname{dim} M$ where $C_{k, j, m}$ is a scalar. Let $j=2 \operatorname{dim} M$. Then the righthand side is 0 , hence $S_{\left(x y^{m+1}\right)}=0$ and $x y^{m+1}=0$. Hence $Y$ acts nilpotently on $A$ and then also on $B$ for each $y \in U$. We now need to show that $U$ (hence $A$ ) acts nilpotently on $B$ and then both assertions in the theorem will follow.

We construct a maximal multiplicative subset $V$ of $U$ such that $V$, and hence its linear span, acts nilpotently on $B$. There are subspaces of $A$ which are the span of multiplicative subsets of $U$ and which act nilpotently on $B$. For example, any single element from $U$ spans such a subspace. From these subspaces, let $W$ be one which is maximal. The multiplicative subsets of $U$ whose span in $W$ have a maximal element $V$ by Zorn's lemma. Now any multiplicative subset $X$ of $U$ which contains $V$ properly cannot be contained in $W$. Then its span contains $W$ properly and, hence, cannot act nilpotently on $B$. Hence $V$ is the desired set.

If $V=U$, then the result holds. Hence suppose that $V \neq U$. Since $V$ acts nilpotently on $B$ it also acts nilpotently on $U$. Hence there exists $k>0$ such that $U V^{[k-1]} \nsubseteq V$ but $U V^{[k]} \subseteq V$. Therefore choose $a \in U V^{[k-1]} \subseteq U$ such that $a \notin V$. Then $a V \subseteq V$. We claim that the multiplicative subset $T=\{a, V\}$ of $U$ acts nilpotently on $B$ which is a contradiction. This is shown by constructing a chain of subspaces of $B$ such that $T$ acts nilpotently on the factors. Since $B V^{[n]}=0$, $W=\{x \in B ; x V=0\} \neq 0$. Now $n(m(a x))=-m(a(x n))-a(x(n m))-x(n(m a))+$ $(a n)(x m)=0$ for all $x \in W$, and all $n, m \in V$. Hence $V(V(a W))=0$ and $V(a W) \subseteq$ $W$. If $V(a W)=0$, then $a W \subseteq W$ and since $V$ annihilates $W, T$ acts nilpotently on $W$. Suppose that $V(a W) \neq 0$. Then $a(m(x a))=-a(a(m x))-x(a(a m))+(m a)(x a)$ shows that $V(a W)$ is $a$-invariant, and since $V$ annihilates $V(a W), T$ acts nilpotently on $V(a W)$. Hence there exists a $T$-invariant subspace $B_{1}$ of $B$ on which $T$ acts nilpotently. Considering $T$ acting on $B / B_{1}$ allows the process to be completed by induction.

COROLlary 1. Let $A$ be a Malcev algebra, $U$ a multiplicative set in $A$ which spans $A$, and $R_{x}$ nilpotent for all $x \in U$. Then $A$ is nilpotent.

Proof. If $Z$ is the center of $A$, then $A / Z$ acts faithfully on $A$. Hence the result follows from the theorem.

Using Corollary 1, several results of Jacobson [7] on automorphisms and derivations of Lie algebras extend to the present case. The proofs also extend, hence are omitted.

COROLlary 2. A Malcev algebra $A$ is nilpotent if it admits one of the following types of operators: 
1. an automorphism of prime period with no nonzero fixed points,

2. an automorphism none of whose characteristic values are roots of unity,

3. a nonsingular derivation when the characteristic of $A$ is 0 .

In the Lie case, the next result is due to D. W. Barnes $[\mathbf{1}]$. The proof is omitted since it is the same as in the Lie algebra case.

Corollary 3. Let $A$ be a Malcev algebra. Then $A$ is nilpotent if and only if each maximal subalgebra of $A$ is an ideal in $A$.

Following the same pattern, a nilpotency characterization dealing with normalizers is obtained even though normalizers are not necessarily subalgebras.

COROLlARY 4. A Malcev algebra is nilpotent if and only if each proper subalgebra is contained properly in its normalizer.

The next corollary has applications to the conjugacy of Cartan subalgebras. It is a result which holds for most of the important classes of algebras which are defined by identities. Let $N$ be an ideal of $A$ and let $U(A, N)$ be the associtive subalgebra of the linear transformations of $A$ which are generated by all $R_{x}, x \in N$.

Corollary 5. Let $N$ be an ideal in $A$ and $U$ a multiplicative set in $N$ such that $R_{x}$ acts nilpotently on $A$ for each $x \in U$, and suppose that $N$ is the linear span of $U$. Then $U(A, N)$ is contained in the radical of $U(A, A)$.

Proof. Let $K$ be the kernel of the natural representation of $N$ acting on $A$. Since $K$ is in the center of $N, K \unrhd N$ and $N / K$ act faithfully on $A$. Hence $N$ acts nilpotently on $A$ by Engel's theorem and $U(A, N)$ is nilpotent. Let $W=\{x \in$ $A ; x U(A, N)=0\} \neq 0$. Now $((x a) m) n=-((n x) a) m-((m n) x) a-((a m) n) x+$ $(m x)(n a)=0$ for all $x \in W, a \in A, n, m \in N$. Hence $N(N(A W))=0$ and $N(A W) \subseteq W$. If $N(A W)=0$, then $A W \subseteq W$ and $W$ is $A$-invariant. Suppose $N(A W) \neq 0$. Then $a(m(x b))=-b(a(m x))-x(b(a m))-m(x(b a))+(m b)(x a) \in$ $(A W) N$ for all $m \in N, b, a \in A$ and $x \in W$. Hence $A(N(W A)) \subseteq N(W A)$ and $N(W A)$ is $A$-invariant. Hence we obtain an $A$-invariant subspace $S_{1}$ of $W$ on which $N$ acts trivially. Working on $A / S_{1}$ yields an $A$-invariant chain $0 \subset S_{1} \subset \cdots \subset S_{k}=$ $A$. Take $\sigma=R_{y_{1}} \cdots R_{y_{j}}$ where at least one $y_{i} \in N$. Then $\sigma$ annihilates each factor and the ideal $D$ of $U(A, A)$ generated by $U(A, N)$ has the same property. Hence $D^{k}=0$ and the result holds.

2. Cartan subalgebras. D. W. Barnes has shown [2] that if $A$ is a selfcentralizing minimal ideal of a solvable Lie algebra $L$, then $A$ is complemented in $L$ and all complements of $A$ are conjugate. This result is quite a useful tool in showing other conjugacy results and a Malcev algebra version would be welcome. However, centralizers of ideals are not necessarily ideals and inner derivations take on a more complicated form in Malcev algebras. Nevertheless, an extension of Barnes' result does exist. In order to show it, some notation is needed. Let $M$ be a Malcev algebra and $A$ an ideal in $M$. Recall that the centralizer of $A$ in $M$ is defined as $C_{M}(A)=\{x \in M: A x=0\}$. For any subalgebra $B$ of $M$, let core $_{M}(B)$ be the largest ideal of $M$ which is contained in $B$. Recall also that $D(a, b)=R_{(a b)}+R_{a} R_{b}-R_{b} R_{a}$ is a derivation of $M$ for all $a, b \in M$. The derivation 
algebra generated by all these $D(a, b)$ is called the algebra of inner derivations. Consider

$$
\exp (D(a, b))=\sum_{n=0}^{\infty} \frac{D(a, b)^{n}}{n !}
$$

which is well defined if $D(a, b)$ is nilpotent and $M$ has characteristic 0 . It is also well defined if $D(a, b)^{n}=0$ for all $n \geq$ characteristic $(M)$. Recall that $J(x, y, z)=$ $(x y) z+(y z) x+(z x) y$ is called the Jacobian.

THEOREM 2. Let $M$ be a solvable Malcev algebra and $A$ a minimal ideal of $M$. Suppose that core ${ }_{M}\left(C_{M}(A)\right)=A$. Then $A$ is complemented in $M$ and all complements of $A$ are conjugate under an automorphism of the form $I+D(x, a)$ where $x \in M$ and $a \in A$.

Proof. Let $B / A$ be a minimal ideal in $M / A$. Thus, $B / A$ is abelian since $M$ is solvable. Then $B A \unrhd M$ by Lemma 4.3 of $[4]$, and $B A \neq 0$ since core ${ }_{M}\left(C_{M}(A)\right)=$ $A$.. Hence $B A=A$ and $B$ is not nilpotent. Hence let $x \in B$ such that $R_{x}$ is not nilpotent. Now $B R_{x} \subseteq A$ and $M\left(R_{x}\right)^{2} \subseteq A$. Hence $M_{0}(x)+A=M$ where $M_{0}(x)$ and $M_{1}(x)$ are the Fitting null and one component of $R_{x}$ acting on $M$. Since $M_{0}(x)$ is a subalgebra of $M$ and $M_{0}(x) \neq M$, it follows that $M_{0}(x) \cap A=0$. Then $A=M_{1}(x)$ and $M_{0}(x)$ is a complement. Let $V$ be another complement to $A$ in $M$. Then $x=c+v, c \in A, v \in V$. Now $x A=A$, hence $x(x A)=A$ and there exists $a \in A$ such that $x(x a)=-c / 2$. Then $x+2 x(x a)=x-c=v$. Now $x D(x, a)=$ $J(x, x, a)+2 x R_{(x a)}=2 x(x a)$, since $J(s, s, t)$ is always 0 . Hence $x(I+D(x, a))=v$. Now $D(x, a)^{2}=0$, hence $I+D(x, a)$ is an automorphism of $M$ which takes $M_{0}(x)$ onto $M_{0}(v)$. Also $v(v V) \subseteq v(v M) \subseteq A$, hence $v(v V) \subseteq A \cap V=0$. Therefore $V \subseteq M_{0}(v)$. But $M_{0}(x) \neq M$, hence $M_{0}(v)$ complements $A$ and $M_{0}(v)=V$.

Let $H$ be a subalgebra of $M . H$ is called a Cartan subalgebra if $H$ is nilpotent and $H$ is the Fitting null component of $H$ acting on $M$. Cartan subalgebras exist in Malcev algebras over an infinite field [9] and in all solvable Malcev algebras [4]. The following three results are shown as in the Lie algebra case, hence the proofs are omitted.

LEMMA 1. Let $H$ be a nilpotent subalgebra of $M . H$ is a Cartan subalgebra if and only if $H=N_{M}(H)$ where $N_{M}(H)$ is the normalizer of $H$ in $M$.

The next two lemmas are extensions of results on Lie algebras due to D. W. Barnes [3].

LEMMA 2. Let $H$ be a Cartan subalgebra of $M$ and $A$ an ideal of $M$. Then $H+A / A$ is a Cartan subalgebra of $M / A$.

LEMMA 3. Suppose $A$ is an ideal of $M$ and $A \subseteq K \subseteq M$. Suppose that $K / A$ is a Cartan subalgebra of $M / A$ and $H$ is a Cartan subalgebra of $K$. Then $H$ is a Cartan subalgebra of $M$.

LEMMA 4. Let $A$ be an abelian ideal of $M$ such that $M / A$ is nilpotent. If $H$ and $K$ are Cartan subalgebras of $M$, then $H$ and $K$ are conjugate under an inner automorphism of $M$ of the form $\exp \left(\sum_{i=1} D\left(x_{i}, a_{i}\right)\right)$ where $x_{i} \in M$ and $a_{i} \in A$ for $i=1, \ldots, s$. 
ProOF. Note that $\exp (D(x, a)) \exp (D(y, b))=\exp (D(x, a)+D(y, b))$ when $x, y \in M$ and $a, b \in A$. Suppose that $B$ is a nonzero ideal of $M$ which is properly contained in $A$. By induction $H+B / B$ and $K+B / B$ are conjugate under $\exp \left(\sum D\left(\bar{x}_{i}, \bar{a}_{i}\right)\right)$. By the note at the beginning of this paragraph, we may assume that $H+B=K+B$. If $H+B=M$, then $0 \neq H \cap A$ is an ideal in $M$. Hence by taking $B \subseteq H \cap A, H=H+B=K+B$. Then $K+B$ is a Cartan subalgebra of $M$, hence $K=K+B$ and $H=K$. Hence we may assume that $H+B \neq M$. Then the result holds by induction.

Now consider the case when $A$ is minimal and $H$ and $K$ are complements of $A$ in $M$. By Theorem 2 the result holds if $J=\operatorname{core}_{M}\left(C_{M}(A)\right)=A$. Hence suppose $J$ contains $A$ properly. Now $J \cap H$ contains a minimal ideal $B$ of $M$. By induction $K+B / B$ is conjugate to $H / B$ under $\exp \left(\sum D\left(\bar{y}_{i}, \bar{c}_{i}\right)\right)$ where $y_{i} \in M, c_{i} \in A$. Then $K+B=H \exp \left(\sum D\left(y_{i}, c_{i}\right)\right)$. Now $K+B$ must be a Cartan subalgebra, hence $K+B=K$ and the result holds.

If $N$ is a nilpotent ideal of $M$, then $U(M, N)$ is a nilpotent ideal of $U(M, M)$ by Corollary 5. Hence $D(m, n)$ is a nilpotent derivation for all $n \in N, m \in$ $M$. If $M$ is a characteristic 0 or if $D(m, n)$ has nilpotency index less than the characteristic of the base field, then $\exp (D(m, n))$ is well defined. Let $I(M, N)$ be the group of all automorphisms generated by all $\exp (D(m, n))$ where $D(m, n)$ satisfies the conditions in the above sentence. We consider the case when $N=M_{w}$, the intersection of the terms in the lower central series of $M$ where the $n$th term $M_{n}$, in the lower central series of $M$, is the space spanned by all products of $n$ elements from $M$. Note that if $A$ is an ideal in $M$, then $A_{w}$ is also an ideal in $M$ since $\left(\left(A_{i} A_{j}\right) A_{k}\right) M \subseteq A_{i+j+k-1}$ is easily verified. Finally if $M$ is solvable and of characteristic 0 , then $M_{w}$ is nilpotent $[\mathbf{9}$, Corollary 3$]$.

THEOREM 3. Let $M$ be a solvable Malcev algebra and let $H$ and $K$ be Cartan subalgebras of $M$. Suppose that if $M$ is of characteristic $p \neq 0$, then $U\left(M, M_{w}\right)^{p-1}$ $=0$. Then $H$ and $K$ are conjugate under $I\left(M, M_{w}\right)$.

Proof. We assume that $M_{w} \neq 0$ and use induction on the dimension of $M$. Let $A$ be a minimal ideal of $M$. Then $H+A / A$ and $K+A / A$ are conjugate under $I\left(M / A,(M / A)_{w}\right)$. Each element of this group is induced by an element of $I\left(M, M_{w}\right)$. Hence we may assume that $H+A=K+A$. If $H+A \neq M$, then $H$ and $K$ are conjugate under $I\left((H+A),(H+A)_{w}\right)$ and the result holds. If $H+A=M$, then $H$ and $K$ are conjugate under $I(M, A)$ by Theorem 2 .

The final results describe relations between Cartan subalgebras and other subalgebras in solvable Malcev algebras.

THEOREM 4. Let $M_{w}$ be abelian. Then $M_{w}$ is complemented in $M$ and all complements are conjugate under an automorphism of the form $\exp \left(\sum D\left(x_{i}, a_{i}\right)\right)$ where $x_{i} \in M$ and $a_{i} \in M_{w}$. The complements of $M_{w}$ are precisely the Cartan subalgebras of $M$.

Proof. Let $H$ be a Cartan subalgebra of $M$ and $M=H+M_{1}$ be the Fitting decomposition of $M$ with respect to $H$. Hence $M_{1}=\sum_{h \in H} M\left(R_{h}\right)^{n}$ where $n=$ $\operatorname{dim} M$. Now $M\left(R_{h}\right)^{n+1}=M\left(R_{h}\right)^{n}$ and $M_{1} H \subseteq M_{1}$ yields that $M_{1} H=M_{1}$. Then $M_{1}\left(R_{H}\right)^{n}=M_{1}$, hence $M_{1} \subseteq M_{w}$ and $M=H+M_{w}$. Now $M_{w}=M M_{w}=$ $\left(H+M_{w}\right) M_{w}=H M_{w}$, hence $M_{w} \subseteq M_{1}$. Therefore $H$ complements $M_{w}$ in $M$. 
Conversely, let $H$ be a complement of $M_{w}$ in $M$. Then $H$ is nilpotent and let $M_{1}$ be the Fitting one component of $H$ acting on $M$. As in the preceding paragraph $M_{1}=M_{w}$, hence $H$ is its own Fitting null component and it is a Cartan subalgebra. The conjugacy part follows from Lemma 4.

Let $M_{w}^{i}$ be the intersection of all terms in the lower central series of $M_{w}^{i-1}$.

THEOREM 5. Suppose that $M_{w}^{n}$ is abelian but not 0 for some $n \geq 1$ and let $H$ be a Cartan subalgebra of $M_{w}^{n-1}$. Let $H_{0}$ be the Fitting null component of $H$ acting on $M$. Then $H_{0}$ is a complement of $M_{w}$ in $M$. Furthermore if $K$ is another complement of $M_{w}^{n}$, then $K$ is the Fitting null component of $J$ acting on $M$ where $J$ is a Cartan subalgebra of $M_{w}^{n-1}$. All complements of $M_{w}^{n}$ in $M$ are conjugate under an inner automorphism of the form $\exp \left(\sum D\left(a_{i}, b_{i}\right)\right)$ where $a_{i} \in M$ and $b_{i} \in M_{w}^{n}$.

ProOF. Let $S=M_{w}^{n-1}$ and $T=M_{w}^{n}$. By Theorem 4, the Cartan subalgebras of $S$ are precisely the complements of $T$ in $S$. For each such $H$, the Fitting null component of $H$ acting on $M$ must complement $T$. If $K$ is another complement of $T$ in $M$, then $J=K \cap S$ complements $T$ in $S$, hence is a Cartan subalgebra of $S$. Clearly the null component of $J$ in $M$ is $K$. Finally since $J$ and $H$ are conjugate in $S$, their null components are conjugate in $M$ under the same inner automorphism.

THEOREM 6. Let $M=N(M)+H$ where $H$ is a subalgebra of $M$ and $N(M)$ is the nilpotent radical of $M$. Then every Cartan subalgebra $K$ of $H$ is of the form $H \cap C$ where $C$ is a Cartan subalgebra of $M$ and $C$ contains the $M$-normalizer of $K$. Furthermore, $C$ is the only Cartan subalgebra of $M$ which contains $K$.

Proof. Let $K$ be a Cartan subalgebra of $H$. For each $x \in M$, let $M_{x}$ be the Fitting null component of $R_{x}$ acting on $M$ and let $S=\bigcap_{x \in K} M_{x}$. Since $K$ is nilpotent, $K \subseteq S$ and $S$ is a subalgebra. We claim that $S$ is a Cartan subalgebra of $M$ and $S \cap H=K$. If $y \neq S$, then there exists $x \in K$ such that $y \notin M_{x}$ and then $x y \notin M_{x}$. Hence $x y \notin S$ and $y \notin N_{M}(S)$. Hence $S=N_{M}(S)$.

Now $K \subseteq S \cap H$ and we assume that this inclusion is proper. Then each $x \in K$ acts nilpotently on the vector space $S \cap H / K$, hence there exists a nonzero subspace of $S \cap H / K$ which is annihilated by $K$. Hence there exists an element of $S \cap H$ which is not in $K$ but is in $N_{M}(K)$, a contradiction. Hence $S \cap H=K$.

Now we show that $S$ is nilpotent. First we claim that $K+N(M)=S+N(M)$. Since $K+N(M) \subseteq S+N(M)$, we assume that the inclusion is proper. Using the same argument as in the preceding paragraph, a contradiction is reached. Hence $K+N(M)=S+N(M)$ and $S=S \cap(K+N(M))=K+(S \cap N(M))$. Now $S \cap N(M)$ is a nilpotent ideal in $S$ and each element of $K$ induces a nilpotent linear transformation of $S$. Hence $S$ is nilpotent by Engel's theorem. Also the $M$-normalizer of $K$ is contained in $S$.

Finally, suppose that $F$ is another Cartan subalgebra of $M$ which contains $K$. Since $F$ is nilpotent, $F \subseteq \bigcap_{x \in K} M_{x}=S$. If $F \neq S$, then $F$ is properly contained in $N_{S}(F)$ since $S$ is nilpotent. But this contradicts $F=N_{M}(F)$. Hence $F=S$.

It is interesting to note the role played by Fitting null components in the last theorems. They are playing the role of the (relative) system normalizers of P. Hall in his classic development of solvable groups $[\mathbf{5}, \mathbf{6}]$. 


\section{REFERENCES}

1. D. W. Barnes, Nilpotency of Lie algebras, Math. Z. 79 (1962), 237-238.

2. __ On the cohomology of solvable Lie algebras, Math. Z. 101 (1967), 242-249.

3. __ On Cartan subalgebras of Lie alebras, Math. Z. 101 (1967), 250-255.

4. A. A. el Malek, On the Frattini subalgebra of a Malcev algebra, Arch. Math. 27 (1981), 306-315.

5. P. Hall, On the Sylow systems of a solvable group, Proc. London Math. Soc. 43 (1937), 316-323.

6. __ On the system normalizers of a solvable group, Proc. London Math. Soc. 43 (1937), 507-528.

7. N. Jacobson, A note on automorphisms and derivations of Lie algebras, Proc. Amer. Math. Soc. 6 (1955), 281-283.

8. __ Lie algebras, Interscience, New York, 1966.

9. E. N. Kuzmin, Malcev algebras and their representations, Algebra and Logic 4 (1968), 48-69.

10. A. A. Sagle, Malcev algebras, Trans. Amer. Math. Soc. 101 (1961), 426-458.

Department of Mathematics, North Carolina State University, Raleigh, NORTH CAROLINA 27650 